\title{
A modification of minimal analytic QCD at low energies
}

\author{
G. Cvetič ${ }^{\mathrm{a}}$, C. Valenzuela ${ }^{\mathrm{a}}$, and I. Schmidt ${ }^{\mathrm{a} *}$ \\ aphysics Department, Universidad Técnica Federico Santa María, Valparaíso, Chile
}

We present an approach which allows for modifications of the (minimal) analytic QCD model of Shirkov, Solovtsov et al.. The discontinuity function of the (minimal) analytic QCD coupling parameter is changed at the low time-like momenta by a simple ansatz motivated by the vector meson dominance for the vector channel of the $e^{+} e^{-}$hadronic decay ratio $R_{V}(s)$. In this way, the additional low energy parameters introduced in the coupling parameter make it possible to obtain the correct value of the semihadronic tau decay ratio without having to introduce the unusually heavy quark masses of $u, d$ and $s$ quarks, in contrast to the minimal analytic QCD. Some numerical results are presented in the leading skeleton approximation for a simple version of such a model. We hope that a more systematic analysis of such models leads to an approximately universal coupling parameter.

It has been well known that the usual perturbative QCD coupling $a_{\mathrm{pt}}\left(Q^{2}\right) \equiv \alpha_{s}\left(Q^{2}\right) / \pi$ (where: $\left.Q^{2}=-q^{2}=-\left(q^{0}\right)^{2}+\vec{q}^{2}\right)$ has nonphysical singularities outside the time-like momentum region, specifically a Landau cut on the space-like axis: $0<Q^{2} \leq \Lambda^{2}$. This can be seen, for example, from the expansion

$$
\begin{aligned}
a_{\mathrm{pt}}\left(Q^{2}\right)= & \frac{1}{\beta_{0} \ln \left(Q^{2} / \Lambda^{2}\right)}-\frac{\beta_{1}}{\beta_{0}^{3}} \frac{\ln \ln \left(Q^{2} / \Lambda^{2}\right)}{\ln ^{2}\left(Q^{2} / \Lambda^{2}\right)} \\
& +\mathcal{O}\left(1 / \ln ^{3}\left(Q^{2} / \Lambda^{2}\right) .\right.
\end{aligned}
$$

Direct application of the Cauchy theorem gives the following dispersion relation for $a_{\mathrm{pt}}$ :

$$
a_{\mathrm{pt}}\left(Q^{2}\right)=\frac{1}{\pi} \int_{\sigma=-\Lambda^{2}}^{\infty} \frac{d \sigma \rho_{1}(\sigma)}{\left(\sigma+Q^{2}\right)}
$$

where $\rho_{1}(\sigma)$ is the (pQCD) discontinuity function of $a_{\mathrm{pt}}$ along the cut axis in the complex $Q^{2}$-plane: $\rho_{1}(\sigma)=\operatorname{Im} a_{\mathrm{pt}}(-\sigma-i \epsilon)$.

The physical observables are, by special relativity and causality, analytic functions of the associated physical momentum squared $q^{2} \equiv-Q^{2}$ in the entire complex $Q^{2}$ plane with the exception of the time-like axis $\left(Q^{2}<0\right)$. Any QCD observable is a function of the invariant coupling $a\left(Q^{2}\right)$, so

\footnotetext{
*The work supported in part by Fondecyt grants No. 1050512 (G.C.), 1030355 (I.S.) and Mecesup grant USA0108 (C.V.). Presented at QCD05, 4-8 July 2005, Montpellier, France.
}

both should have the same analyticity properties. Therefore, the cut sector $0<Q^{2} \leq \Lambda^{2}$ in $a_{\mathrm{pt}}\left(Q^{2}\right)$, Eqs. (11)-(2), is nonphysical. A simple analytization, which is minimal in a sense, is obtained by eliminating the unphysical cut in the dispersion relation (2) while keeping elsewhere the same discontinuity function $\rho_{1}(\sigma)$, thus leading to a specific analytic coupling $\mathcal{A}_{1}\left(Q^{2}\right)$ [1]

$$
\mathcal{A}_{1}\left(Q^{2}\right)=\frac{1}{\pi} \int_{\sigma=0}^{\infty} \frac{d \sigma \rho_{1}(\sigma)}{\left(\sigma+Q^{2}\right)} .
$$

Calculational aspects of this prescription were investigated in 2. Other approaches to analytization of $a_{\mathrm{pt}}$ have focused on the analyticity properties of the beta function 34, or the addition of power correction terms $1 /\left(Q^{2}\right)^{n}$ to $\mathcal{A}_{1}$ [5]. Our approach considers a modification of the pQCD discontinuity function $\rho_{1}(\sigma)$ of Eq. (3) in the $\sigma \sim \Lambda$ region of the time-like axis, resulting in a different analytic coupling $\overline{\mathcal{A}}_{1}\left(Q^{2}\right)$

$$
\overline{\mathcal{A}}_{1}\left(Q^{2}\right)=\frac{1}{\pi} \int_{\sigma=0}^{\infty} \frac{d \sigma \bar{\rho}_{1}(\sigma)}{\left(\sigma+Q^{2}\right)} .
$$

We recall that relation (4) defines the coupling in the entire complex $Q^{2}$-plane except on the timelike axis $Q^{2}<0$. Then, in the analytic approach the coupling on the time-like axis $s=-Q^{2}>0$ can be defined in the following convenient way [6] 


$$
\overline{\mathfrak{A}}_{1}(s)=\frac{i}{2 \pi} \int_{-s+i \epsilon}^{-s-i \epsilon} \frac{d \sigma^{\prime}}{\sigma^{\prime}} \overline{\mathcal{A}}_{1}\left(\sigma^{\prime}\right)
$$

where the integration is in the $Q^{2} \equiv \sigma^{\prime}$ plane avoiding the (time-like) cut $\sigma^{\prime}<0$. This relation between $\overline{\mathcal{A}}_{1}\left(Q^{2}\right)$ and $\overline{\mathfrak{A}}_{1}(s)$ is the same as the one between the (vector channel) Adler function $D_{V}\left(Q^{2}\right)$ and the $e^{+} e^{-}$hadronic scattering cross section ratio $R_{V}(s)$. In addition to relations (4) and (5), the following relations hold [7]:

$$
\begin{aligned}
\overline{\mathfrak{A}}_{1}(s) & =\frac{1}{\pi} \int_{s}^{\infty} \frac{d \sigma}{\sigma} \bar{\rho}_{1}(\sigma), \\
\overline{\mathcal{A}}_{1}\left(Q^{2}\right) & =Q^{2} \int_{0}^{\infty} \frac{d s \overline{\mathfrak{A}}_{1}(s)}{\left(s+Q^{2}\right)^{2}}, \\
\frac{d}{d \ln \sigma} \overline{\mathfrak{A}}_{1}(\sigma) & =-\frac{1}{\pi} \bar{\rho}_{1}(\sigma) .
\end{aligned}
$$

The value of the coupling parameter at the origin is in general finite $\left(\overline{\mathcal{A}}(0)=\overline{\mathfrak{A}}_{1}(0)=\right.$ $\left.(1 / \pi) \int_{0}^{\infty} d \sigma \bar{\rho}_{1}(\sigma) / \sigma.\right)$

The minimal analytic QCD model 16 [cf. Eq. (3)] contains only one free parameter the scale $\Lambda$ contained in the pQCD discontinuity function $\rho_{1}(\sigma)$. By fitting the measured values of high energy QCD observables (at $Q$ or $\sqrt{s} \gtrsim 10$ $\mathrm{GeV})$ to the model, the value $\Lambda(\overline{\mathrm{MS}})_{n_{f}=5} \approx$ $0.26 \mathrm{GeV}$ was obtained 7, corresponding to $\Lambda(\overline{\mathrm{MS}})_{n_{f}=3} \approx 0.4 \mathrm{GeV}$. At lower energies, however, a problem appeared. The $V$-channel of the semihadronic $\tau$ decay ratio, without the strangeness production, has been measured with high precision by the ALEPH and OPAL [8] Collaborations with the result: $R_{\tau, V}(\triangle S=0) \approx 1.19$, and where this quantity has been normalized so that in pQCD: $R_{\tau, V}(\triangle S=0)=\left[1+a_{\mathrm{pt}}+\mathcal{O}\left(a_{\mathrm{pt}}^{2}\right)\right]$. However, fitting this value with the prediction of the minimal analytic QCD gave: $\Lambda(\overline{\mathrm{MS}})_{n_{f}=3} \approx$ $0.8 \mathrm{GeV}$ [9]. This problem was then solved by the same authors in Ref. [10, where the value $\Lambda(\overline{\mathrm{MS}})_{n_{f}=3} \approx 0.4 \mathrm{GeV}$ was obtained at the price of introducing large quark masses $m_{u} \approx m_{d} \approx$ $0.25 \mathrm{GeV}$ which implied very strong threshold effects in the quantity $R_{\tau, V}(\triangle S=0)$. The introduction of (effective) heavy quark masses may be regarded as unattractive or hard to justify theoretically.
A different possibility would be to include (some) nonperturbative effects within the timelike coupling $\mathfrak{A}_{1}(s)\left[\Leftrightarrow \mathcal{A}_{1}\left(Q^{2}\right)\right]$. For that purpose let us consider first the isovector hadronic spectral function $R_{V}(s)$ [with the normalization $\left.R_{V}(s)=1+a_{\mathrm{pt}}+\mathcal{O}\left(a^{2}\right)\right]$ which has been measured with high precision [8]. At low energies $\sqrt{s} \lesssim 1$ $\mathrm{GeV}$ it is dominated by the $\rho$-meson resonance $\left(M_{\rho}=0.776 \mathrm{GeV}\right)$. On the other hand, the Adler function is obtained by the relation

$$
D_{V}\left(Q^{2}\right)=Q^{2} \int_{0}^{\infty} \frac{d s R_{V}(s)}{\left(s+Q^{2}\right)^{2}}
$$

which is analogous to Eq. (7). If in the aforementioned resonance region we use a narrow width approximation (NWA) for $R_{V}(s)$, in the spirit of the Vecton Meson Dominance (VMD), then

$$
\begin{gathered}
\left(4 \pi^{2}\right)^{-1} R_{V}(s)_{\mathrm{VMD}}=2 f_{\rho}^{2} M_{\rho}^{2} \delta\left(s-M_{\rho}^{2}\right) \\
+\left(4 \pi^{2}\right)^{-1} R_{V}(s)_{\mathrm{pQCD}} \theta\left(s-M_{\mathrm{cut}}^{2}\right),
\end{gathered}
$$

where $f_{\rho}^{2}=\left(f_{\rho}^{2}\right)_{\mathrm{NWA}} \approx 0.0305$, and $\theta(x)$ is the step function $(+1$ for $x>0$, zero for $x<0)$. The cut value $M_{\text {cut }}^{2} \approx 1.53 \mathrm{GeV}^{2}$ [1] was fixed so that the corresponding Adler function (9) is closest to the "experimental" Adler function $D_{V}\left(Q^{2}\right)_{\exp }$ [obtained via relation (9) from the measured $\left.R_{V}(s)_{\exp }\right]$ in the low $Q^{2}$ sector $\left(0 \leq Q^{2}<2 \mathrm{GeV}^{2}\right)$ where the quark masses can presumably be neglected. The agreement of the two Adler functions is then remarkably close there. Since in the analytic QCD $R_{V}(s)=1+\overline{\mathfrak{A}}_{1}(s)+\mathcal{O}\left(\left(\overline{\mathfrak{A}}_{1}\right)^{2}\right)$, then the VMD form (10) suggests

$$
\begin{aligned}
& \overline{\mathfrak{A}}_{1}(s)=\left[R_{V}(s)-1\right]+\mathcal{O}\left(\left(\overline{\mathfrak{A}}_{1}\right)^{2}\right) \\
& \quad \approx\left[c_{f} M_{r}^{2} \delta\left(s-M_{r}^{2}\right)-1\right] \\
& \quad+\left(\mathfrak{A}_{1}(s)+1\right) \theta\left(s-M_{0}^{2}\right)+\mathcal{O}\left(\left(\overline{\mathfrak{A}}_{1}\right)^{2}\right),
\end{aligned}
$$

where $\mathfrak{A}_{1}(s)$ is the minimal analytic QCD timelike coupling (containing the parameter $\Lambda$ ); $c_{f}$, $M_{r}$ and $M_{0}$ are additional parameters whose values are comparable to $8 \pi^{2} f_{\rho}^{2}, M_{\rho}$ and $M_{\text {cut }}$ of Eq. (10), respectively. Neglecting higher order terms in (11) gives

$\overline{\mathfrak{A}}_{1}(s)=\left\{\begin{array}{cc}{\left[c_{f} M_{r}^{2} \delta\left(s-M_{r}^{2}\right)-1\right]} & \left(s<M_{0}^{2}\right) \\ \mathfrak{A}_{1}(s) & \left(s \geq M_{0}^{2}\right)\end{array}\right\}$

and $M_{r}<M_{0}$ is implicitly assumed. Applying the transformation (7) to this VMD-motivated 
time-like coupling, the corresponding analytic space-like coupling is obtained

$$
\begin{aligned}
\overline{\mathcal{A}}_{1}\left(Q^{2}\right)= & c_{f} \frac{M_{r}^{2} Q^{2}}{\left(Q^{2}+M_{r}^{2}\right)^{2}}-d_{f} \frac{M_{0}^{2}}{\left(Q^{2}+M_{0}^{2}\right)} \\
& +\frac{1}{\pi} \int_{\sigma=M_{0}^{2}}^{\infty} \frac{d \sigma \rho_{1}(\sigma)}{\left(\sigma+Q^{2}\right)}
\end{aligned}
$$

where

$$
d_{f}=1+\frac{1}{\pi} \int_{\sigma=M_{0}^{2}}^{\infty} \frac{d \sigma}{\sigma} \rho_{1}(\sigma)
$$

The presented version of the model contains four parameters: $c_{f}, M_{r}, M_{0}$, and $\Lambda$ (the latter is contained in $\mathfrak{A}_{1}(s)$ and in $\left.\rho_{1}(s)\right)$. In general, analytic coupling (13) differs from the minimal analytic coupling $\mathcal{A}_{1}\left(Q^{2}\right)$, Eq. (3), by terms $\sim\left(\Lambda^{2} / Q^{2}\right)$.

In this presentation we choose, for simplicity, the additional condition that the two couplings virtually merge at high momenta, i.e., that $\overline{\mathcal{A}}_{1}\left(Q^{2}\right)-\mathcal{A}_{1}\left(Q^{2}\right) \sim\left(\Lambda^{2} / Q^{2}\right)^{2}$. Then, (a) the high energy QCD observables $(Q, \sqrt{s} \gtrsim 10 \mathrm{GeV})$ give the value $\Lambda_{n_{f}=3} \approx 0.4 \mathrm{GeV}$ as in the minimal analytic model [7, and (b) an additional relation is obtained

$$
\begin{aligned}
& M_{r}^{2} c_{f}=\frac{1}{\pi} \int_{0}^{M_{0}^{2}} d \sigma \rho_{1}(\sigma)+ \\
&+M_{0}^{2}\left[\frac{1}{\pi} \int_{M_{0}^{2}}^{\infty} \frac{d \sigma}{\sigma} \rho_{1}(\sigma)+1\right] .
\end{aligned}
$$

Therefore, in such a case the model contains two yet undetermined parameters, the scales $M_{r}$ and $M_{0}$, i.e., $\overline{\mathcal{A}}_{1}=\overline{\mathcal{A}}_{1}\left(Q^{2} ; M_{r}, M_{0}\right), \overline{\mathfrak{A}}_{1}=$ $\overline{\mathfrak{A}}_{1}\left(s ; M_{r}, M_{0}\right)$. These two parameters can then be fixed, for example, by the condition that the measured semihadronic decay value $R_{\tau, V}(\triangle S=$ $0)$ and a "measured" value of the $V$-channel Adler function $D_{V}\left(Q^{2}\right)$ (at a given chosen low $Q^{2}$ ) be reproduced in the model. In this presentation, we will use the approximation of the leading skeleton (l.s.), also known as large- $\beta_{0}$ resummation. Ac- cording to this resummation 1213

$$
\begin{aligned}
& D_{V}\left(Q^{2}\right)^{(1 . s .)}= \\
& \quad 1+\frac{1}{4} \int_{0}^{\infty} d t w_{D}(t) a_{\mathrm{pt}}\left(t Q^{2} e^{-5 / 3}\right), \\
& R_{\tau, V}(\triangle S=0)^{(1 . \mathrm{s} .)}= \\
& \quad 1+\frac{1}{4} \int_{0}^{\infty} d t W_{\tau}(t) a_{\mathrm{pt}}^{(\mathrm{t} .1 .)}\left(t m_{\tau}^{2} e^{-5 / 3}\right),
\end{aligned}
$$

where the scheme invariant distribution functions $t w_{D}(t)$ and $t W_{\tau}(t)$ are explicitly known 1213 (cf. also 14) and are peaked at around $t \sim 1$. The factor $e^{-5 / 3}$ in the above formulas means that the $\overline{\mathrm{MS}}$ scheme is used for $a_{\mathrm{pt}}$, i.e., $\Lambda_{\overline{\mathrm{MS}}}$ appears there. In Eq. (17), $a_{\mathrm{pt}}^{(\mathrm{t} . \mathrm{l} .)}$ is a time-like coupling [13. The expressions (16) and (17) have renormalon ambiguities and the time-like analytic continuation ambiguities in pQCD, both manifested via the existence of unphysical singularities of $a_{\mathrm{pt}}$ on the Landau cut. In our model, there is no Landau cut, thus no ambiguities, and $a_{\mathrm{pt}}$ in the above expressions gets replaced by the space-like and the time-like couplings, respectively

$$
\begin{aligned}
& D_{V}\left(Q^{2}\right)^{(1 . s .)}= \\
& \quad 1+\frac{1}{4} \int_{0}^{\infty} d t w_{D}(t) \overline{\mathcal{A}}_{1}\left(t Q^{2} e^{-5 / 3}\right), \\
& R_{\tau, V}(\triangle S=0)^{(1 . \mathrm{ss})}= \\
& \quad 1+\frac{1}{4} \int_{0}^{\infty} d t W_{\tau}(t) \overline{\mathfrak{A}}_{1}\left(t m_{\tau}^{2} e^{-5 / 3}\right) .
\end{aligned}
$$

To fix the two parameters $M_{r}$ and $M_{0}$, we choose as experimental inputs the values

$$
\begin{aligned}
D_{V}(Q=0.7 \mathrm{GeV}) & =1.00, \\
R_{\tau, V}(\triangle S=0) & =1.196 .
\end{aligned}
$$

The value (20) was obtained in Ref. 11] on the basis of the ALEPH measurements of $R_{V}(s)$ [8], by applying relation (9) and neglecting the light quark masses. The value (21) is based on the measurements of the ALEPH and OPAL Collaborations [8], but with (small) quark mass effects subtracted out (cf. also [15]). Requiring that the leading skeleton expressions (18) and (19) reproduce the values (20) and (21), respectively, gives values $M_{r}=0.428 \mathrm{GeV}$ and $M_{0}=0.742 \mathrm{GeV}$ in the $\overline{\mathrm{MS}}$ scheme (this corresponds in the $V$ scheme 
to $M_{r}=0.985 \mathrm{GeV}$ and $M_{0}=1.708 \mathrm{GeV}$ ). With these values of the scale parameters, we present in Fig. 1 the curve for $D_{V}\left(Q^{2}\right)$ as function of $Q$ as predicted by the model, in the leading skeleton approximation (18), and the "experimental" curve [1] as obtained by the relation (9) (in the massless quark limit) on the basis of the measured values of $R_{V}(s)$. We see that the two curves agree reasonably well in the range $Q \lesssim 1 \mathrm{GeV}$. At higher values of $Q$, the effects of the $c$ quark mass become important [16. In our calculation we used for $\rho_{1}(\sigma) \equiv \operatorname{Im} a_{\mathrm{pt}}(-\sigma-i \epsilon)$ the expansion (11) at the three-loop level [i.e., including terms $\sim \ln ^{3}(Q / \Lambda)$.]

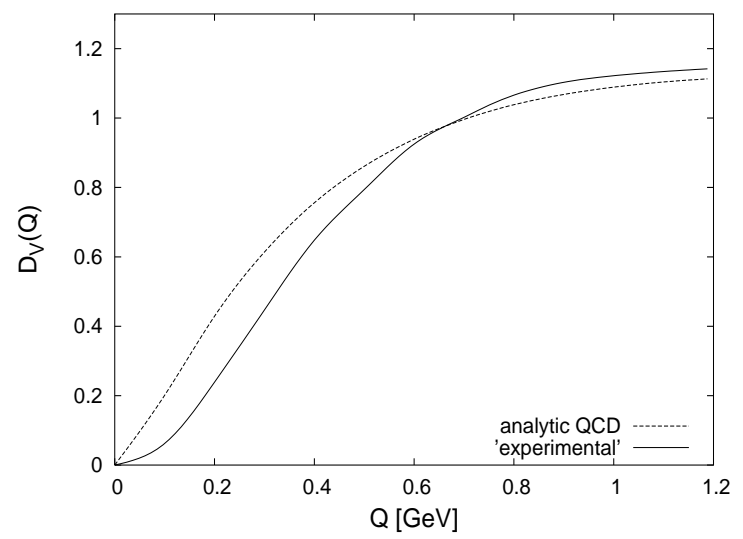

Figure 1. The "experimental" curve for $D_{V}(Q)$ [1], and the prediction of our simple model of $V M D$-motivated analytic $Q C D$ in the leading skeleton approximation.

We see that the model, even in the leading skeleton approximation and in one of its simplest forms as presented here, reproduces reasonably well the low energy behavior of the Adler function, and the measured semihadronic $\tau$ decay ratio $R_{\tau}$, while at the same time the quark masses are set equal zero. We recall that the minimal analytic QCD requires large effective quark masses to reproduce the correct value of $R_{\tau}$ [10.

In general, at low energies the quark mass effects cannot be entirely neglected. Further, chiral symmetry breaking, responsible among other things for a nonzero pion mass, brings important contributions to some low energy QCD observables, such as the axial channel $R_{A}(s)$ and
$D_{A}\left(Q^{2}\right)$. Such effects will eventually have to be taken into account in the framework of the models suggested here. Furthermore, the contributions beyond the leading skeleton have to be considered, possibly by analytization of the power terms $\left(a_{\mathrm{pt}}\left(Q^{2}\right)\right)^{n}(n \geq 2)$ in the spirit of the approach of [1. The type of models suggested here can be implemented in a more sophisticated way than the simplest version presented here - e.g., by going beyond the simple VMD-motivated delta ansatz for the low energy coupling $\overline{\mathfrak{A}}_{1}(s)$.

\section{REFERENCES}

1. D. V. Shirkov and I. L. Solovtsov, hep-ph/9604363 Phys. Rev. Lett. 79, 1209 (1997).

2. D. S. Kurashev and B. A. Magradze, Theor. Math. Phys. 135, 531 (2003); hep-ph/0104142 A. I. Alekseev, Few Body Syst. 32, 193 (2003) hep-ph/0211339.

3. A. V. Nesterenko, Phys. Rev. D 62, 094028 (2000); Phys. Rev. D 64, 116009 (2001); Int. J. Mod. Phys. A 18, 5475 (2003); A. V. Nesterenko and J. Papavassiliou, Phys. Rev. D 71, 016009 (2005).

4. P. A. Rączka, presented at QCD05, Montpellier, July 2005.

5. A. I. Alekseev, hep-ph/0503242

6. K. A. Milton and I. L. Solovtsov, Phys. Rev. D 55, 5295 (1997).

7. D. V. Shirkov, Eur. Phys. J. C 22, 331 (2001); and also hep-ph/0408272 (presented at QCD04, Montpellier, July 2004).

8. R. Barate et al. [ALEPH Collaboration], Eur. Phys. J. C 4, 409 (1998); K. Ackerstaff et al. [OPAL Collaboration], Eur. Phys. J. C 7, 571 (1999).

9. K. A. Milton, I. L. Solovtsov and O. P. Solovtsova, Phys. Lett. B 415, 104 (1997); K. A. Milton, I. L. Solovtsov, O. P. Solovtsova and V. I. Yasnov, Eur. Phys. J. C 14, 495 (2000).

10. K.A. Milton, I.L. Solovtsov, O.P. Solovtsova, Phys. Rev. D 64, 016005 (2001).

11. S. Peris, M. Perrottet and E. de Rafael, JHEP 9805, 011 (1998).

12. M. Neubert, Phys. Rev. D 51, 5924 (1995). 
13. M. Neubert, hep-ph/9502264

14. P. Ball, M. Beneke and V. M. Braun, Nucl. Phys. B 452, 563 (1995).

15. G. Cvetič and T. Lee, Phys. Rev. D 64, 014030 (2001); G. Cvetič, C. Dib, T. Lee and I. Schmidt, Phys. Rev. D 64, 093016 (2001).

16. S. Eidelman, F. Jegerlehner, A. L. Kataev and O. Veretin, Phys. Lett. B 454, 369 (1999). 